\title{
Keratocystic Odontogenic Tumor
}

\author{
Elizabeth A. Grasmuck • Brenda L. Nelson
}

Received: 21 September 2009/Accepted: 15 October 2009/Published online: 10 November 2009

(c) Humana 2009

\begin{abstract}
The keratocystic odontogenic tumor is a benign developmental tumor with many distinguishing clinical and histologic features. These characteristics are reviewed in the setting of a typical presentation. The newly acknowledged neoplastic potential and its implications for treatment strategies are also discussed.
\end{abstract}

\section{History}

A 20-year-old male presented for a routine dental exam with no complaints. Panoramic radiograph films were performed in accordance with standard procedures. A radiolucent lesion was identified in the right maxilla and presumed to be a dentigerous cyst. With this working diagnosis, the lesion was curetted and submitted for microscopic evaluation.

\section{Radiographic Features}

The panoramic radiograph revealed a large radiolucent lesion of the right maxilla with well-defined, smooth, corticated margins. The radiolucency was associated with an impacted third molar, displacing it superiorly toward the

Disclaimer The opinions and assertions expressed herein are those of the author and are not to be construed as official or representing the views of the Department of the Navy or the Department of Defense.

E. A. Grasmuck $(\bowtie) \cdot$ B. L. Nelson

Department of Anatomic Pathology, Naval Medical Center San

Diego, 34800 Bob Wilson Drive, San Diego,

CA 92134-5000, USA

e-mail: elizabeth.grasmuck@med.navy.mil right lower orbital rim. Directly inferior to the lesion there was an opacification of the maxillary sinus (Fig. 1).

\section{Diagnosis}

Histological examination of hematoxylin and eosin stained slides demonstrated a fibrous cyst wall with a uniform stratified squamous epithelium, six to eight cells in thickness. The epithelium was distinctive for a layer of columnar, pallisading, hyperchromatic basal cells (Fig. 2). Rete ridges were absent and focally the epithelium was detached from the underlying fibrous tissue. The luminal surface was parakeratotic with a corrugated appearance. The lumen contained keratinaceous and cellular debris (Fig. 3).

\section{Discussion}

The keratocystic odontogenic tumor (KCOT), formerly known as the odontogenic keratocyst (OKC), received its new designation in order to better convey its neoplastic nature [1]. It is a benign developmental odontogenic tumor with many distinguishing clinical and histologic features. Among them are: a potential for locally destructive behavior, a relatively high recurrence rate, and designation as a consistent finding in the nevoid basal cell carcinoma syndrome, or Gorlin syndrome.

The keratocystic odontogenic tumor is believed to arise from dental lamina and represents between $4-12 \%$ of all odontogenic cysts. The lesion occurs over a wide age range with a peak in the second and third decades and demonstrates a predilection for white males [2]. The mandible is more commonly involved than the maxilla $(65-83 \%$ of 


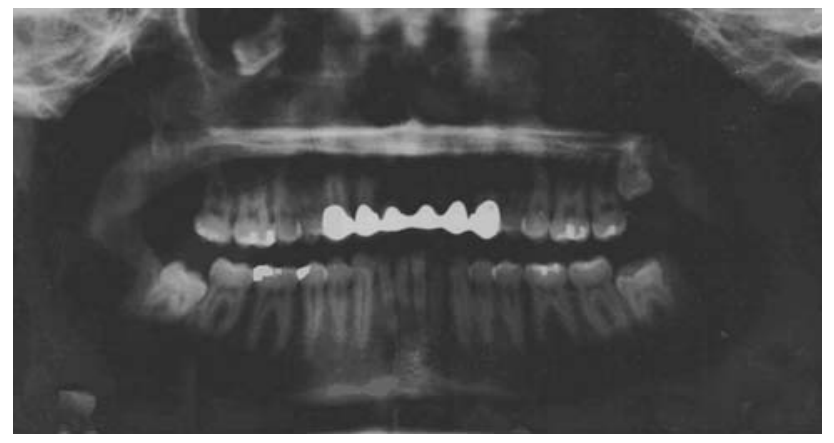

Fig. 1 Panographic film shows a radiolucent lesion of the right maxilla and an associated impacted maxillary third molar. Radiographically this cannot be differentiated from a dentigerous cyst

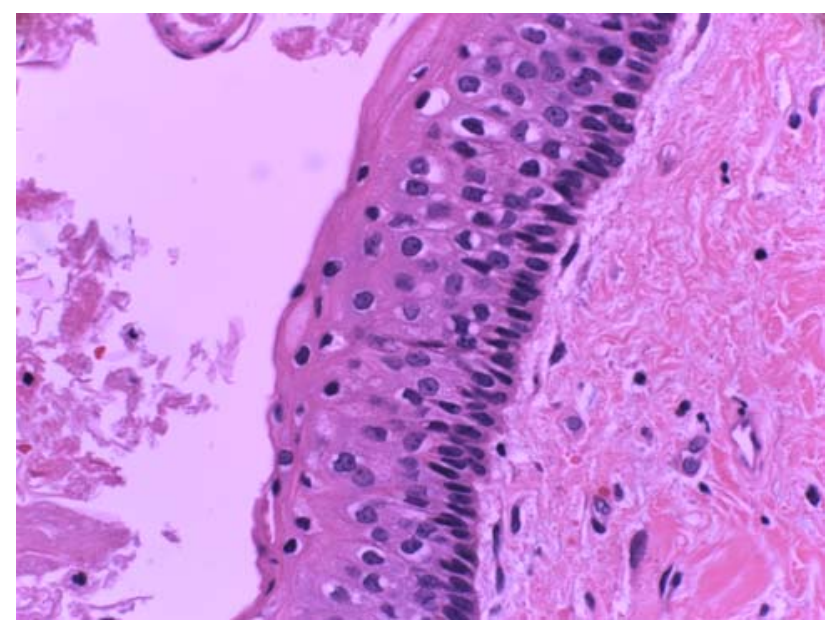

Fig. 2 Hematoxylin and eosin shows a high power view of the palisaded, hyperchromatic basal layer

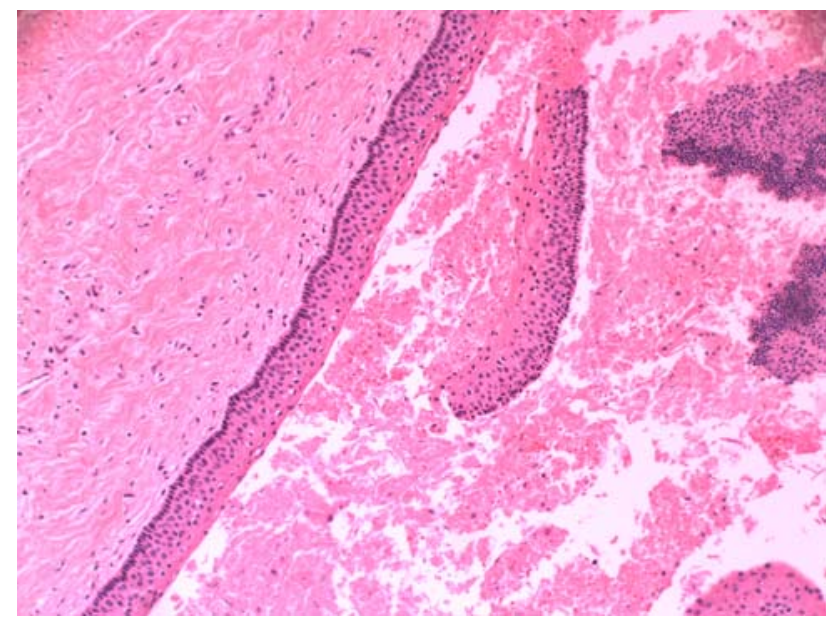

Fig. 3 Hematoxylin and eosin shows an epithelium lining that is 6-8 cells thick. Keratinaceous debris is seen closely apposed to the wavy parakeratotic surface cases) with roughly one-half originating at the angle of the mandible [1]. While the KCOT is generally regarded as an intraosseous lesion, rare peripheral cases have been reported. The majority of cases involve the gingival or alveolar mucosa in the canine-premolar region [3].

Most keratocystic odontogenic tumors are discovered incidentally during review of routine dental radiographs. Occasionally, pain, swelling, and drainage will herald a secondary infection of the cyst. Imaging studies generally show unilocular radiolucencies with well-demarcated sclerotic margins. Larger lesions may become multi-loculated with scalloped borders. The cyst is often times associated with an impacted tooth, and is thought to be a dentigerous cyst. Adjacent teeth may be displaced, but root resorption rarely occurs. CT scans and contrast-enhanced MRI may be useful in assessment of cortical perforation and soft tissue involvement [1, 2, 4].

The gross findings of keratocytic odontogenic tumors are a thin fibrous wall that is usually collapsed. When the cyst is received intact, however, the lumen may be filled with either clear fluid, yellow-white keratin, or an unerupted tooth. The classic histopathologic features of the KCOT are distinctive, but may be altered by inflammation. The uniformly thin epithelial lining of six to eight cells does not demonstrate rete ridges and may result in the epithelium lifting from the underlying fibrous connective tissue. This cleft is a microscopic feature that is considered artifactual but characteristic of the KCOT. The epithelium is also distinctive for a well-defined, pallisaded basal layer of hyperchromatic columnar to cuboidal cells. The luminal surface displays wavy parakeratotic epithelial cells and is frequently described as corrugated. Finally, the cystic cavity may contain keratinacious material [1, 2, 4].

A consensus has been obtained regarding the keratocystic odontogenic tumor's potential for aggressive behavior and it's classification as a neoplasm. The gene associated with the Gorlin Syndrome (9q22.3-q31) likely functions as a tumor suppressor. Studies of syndromic and sporadic KCOT have provided evidence of a two-hit mechanism in the pathogenesis of many of these tumors involving allelic loss at two or more loci of 9q22 leading to overexpression of bcl-1 and TP53 [1]. Additional markers of proliferation, PCNA and Ki67 have also been found to be expressed more strongly in KCOT's than other odontogenic cysts [5].

Multiple treatments for the keratocystic odontogenic tumor have been proposed and debated. The challenge lies in minimizing both the risk of recurrence and morbidity of an extensive resection. Numerous modalities ranging from decompression alone, to simple enucleation with or without curettage, to resection have been employed in the management of KCOT [6]. Researchers have attempted to systematically investigate the outcomes of these procedures, however integrating data across individual studies 
revealed too many inconsistencies to draw definitive conclusions. The likelihood of recurrences and the ability to study theses outcomes statistically is affected by numerous variables including: subtle discrepancies in treatments, differences in the locations of the cysts, presence or absence of infection, associated teeth, involvement of mucosa, size of the lesion, and association with the Gorlin Syndrome [7]. Because of these numerous factors, the treatment and prognosis of keratocystic odontogenic tumors must be managed in a case by case manner. While the cyst is a benign neoplasm, its recurrence rate may be as high as $17-56 \%$ with simple enucleation. If an adjunctive treatment is added, such as the application of Carnoy's solution or decompression before enucleation, the recurrence rate is reported to be between 1 and $8.7 \%$. Finally, resection is uniformly reported to result in essentially no recurrences, but may be unacceptably extensive given the benign nature of the disease. Further prospective studies may provide greater insight into the prognostic implications of this intriguing entity [7].

\section{References}

1. Barnes L, Eveson JW, Reichart P, Sidransky D, editors. World health organization classification of tumours: pathology and genetics of head and neck tumours. Lyon: IARC Press; 2005.

2. Thompson L, Goldblum J, editors. Head and neck pathology; a volume in the series foundations in diagnostic pathology. Philadelphia: Elsevier; 2006.

3. Chi A, Owings J, Muller S. Peripheral odontogenic keratocyst: report of two cases and review of the literature. Oral Surg Oral Med Oral Pathol Oral Radiol Endod. 2005;99:71-8.

4. Press S. Odontogenic tumors of the maxillary sinus. Curr Opin Otolaryngol Head Neck Surg. 2008;16:47-54.

5. Shear M. The aggressive nature of the odontogenic keratocyst: is it a benign cystic neoplasm? Part 2 proliferation and genetic studies. Oral Onco. 2002;38:323-331.

6. Giuliani M, Grossi G, Lajolo C, Bisceglia M, Herb K. Conservative management of a large odontogenic keratocyst: report of a case and review of the literature. J Oral Maxillofac Surg. 2006; 64:308-16.

7. Blanas N, Freund B, Schwartz M, Furst I. Systematic Review of treatment and prognosis in the odontogenic keratocyst. Oral Surg Oral Med Oral Pathol Oral Radiol Endod. 2000;90:553-8. 